Sociologie et sociétés

\title{
Jeunes musulmans et citoyenneté culturelle : retour sur des expériences de recherche en Afrique de l'Ouest francophone
}

\section{Marie-Nathalie Leblanc et Muriel Gomez-Perez}

Volume 39, numéro 2, automne 2007

Sociétés africaines en mutation : entre individualisme et communautarisme

Mutating African Societies: Between Individualism and

Communitarism

URI : https://id.erudit.org/iderudit/019083ar

DOI : https://doi.org/10.7202/019083ar

Aller au sommaire du numéro

Éditeur(s)

Les Presses de l'Université de Montréal

ISSN

0038-030X (imprimé)

1492-1375 (numérique)

Découvrir la revue

Citer cet article

Leblanc, M.-N. \& Gomez-Perez, M. (2007). Jeunes musulmans et citoyenneté culturelle : retour sur des expériences de recherche en Afrique de l'Ouest francophone. Sociologie et sociétés, 39(2), 39-59.

https://doi.org/10.7202/019083ar
Résumé de l'article

La question des jeunes est un élément essentiel pour comprendre les dynamiques de l'espace public et les pratiques citoyennes qui émergent dans l'Afrique d'aujourd'hui. Leur importance tient non seulement à leur poids démographique imposant, mais aussi à leur transformation, souvent dramatique, en tant qu'acteurs sociaux dans l'espace public africain au cours des années 1990. Cette transformation encourage la création de nouvelles formes de légitimité et de nouveaux espaces d'expression individuelle ou collective, et correspond à une mutation radicale de l'idée de citoyenneté, qui fait appel à de nouvelles ressources et qui remodèle les dynamiques nationales d'inclusion et d'exclusion. Ainsi, la constitution de nouveaux espaces d'expression encourage une conception moins restrictive de la participation citoyenne dans la mesure où les jeunes veulent faire entendre leur opinion et participer ouvertement aux divers débats de société. En lien avec les concepts de " citoyenneté culturelle " et de " contre-nation ", nous nous proposons dans cet article d'examiner le rôle des jeunes dans le contexte de réaffirmation de l'identité islamique qui a marqué l'Afrique de l'Ouest francophone à partir des années 1980 et plus encore dans les années 1990, particulièrement dans les grands centres urbains du Sénégal, du Mali, du Burkina Faso et de la Côte d'Ivoire. Nous nous penchons plus spécifiquement sur la question du rapport entre jeunes (comme catégorie sociale), religion et espace public.
Tous droits réservés @ Les Presses de l’Université de Montréal, 2008
Ce document est protégé par la loi sur le droit d'auteur. L’utilisation des services d'Érudit (y compris la reproduction) est assujettie à sa politique d'utilisation que vous pouvez consulter en ligne.

https://apropos.erudit.org/fr/usagers/politique-dutilisation/ 


\section{Jeunes musulmans et citoyenneté culturelle: retour sur des expériences de recherche en Afrique de l'Ouest francophone}

MARIE-NATHALIE LEBLANC

Professeure

Département de sociologie

Université du Québec à Montréal

C. P. 8888 , succursale Centre-ville

Montréal (Québec) $\mathrm{H}_{3} \mathrm{C}_{3} \mathrm{P} 8$

Leblanc.marie-nathalie@uqam.ca

MURIEL GOMEZ-PEREZ

Professeure

Pavillon Charles-De Koninck

Université Laval

Département d'histoire

Québec (Québec) G1K 7P4

Muriel.gomez-perez@hst.ulaval.ca

À noter, le présent texte est quelque peu différent de la version imprimée.

Il est essentiel d'aborder la question des jeunes pour comprendre les dynamiques de l'espace public ${ }^{1}$ et les pratiques citoyennes qui émergent dans l'Afrique d'aujourd'hui. Cette importance tient non seulement à leur poids démographique imposant ${ }^{2}$, mais aussi à leur transformation, souvent dramatique, en acteurs sociaux dans l'espace public africain au cours des années $1990^{3}$. L'insertion des jeunes dans l'espace public

1. Défini comme un espace plus ou moins libre encourageant le discours et l'échange d'idées contradictoires, mais aussi l'expression non verbale (voir Diouf, 2003, pour une définition similaire de l'espace public).

2. Selon le contexte national, de 40 à $60 \%$ des Africains ont moins de 14 ans.

3. Pour une description empirique de l'apparition des jeunes en tant que catégorie sociale d'acteurs sociaux en Afrique de l'Ouest, voir entre autres Diouf, 2003; Jua, 2003; Maxwell, 2001; Meyer, 1998; Abdullah et al., 1997; Cruise O’Brien, 1996; Richards, 1996; El-Kenz, 1995. 
reste liée à leur exclusion des centres du pouvoir économique et politique. Cette question, selon Diouf et Collignon, «n'est visible, perceptible, que lorsqu'elle devient objet de controverses dans l'espace public et/ou de perturbations de l'ordre public dans ses registres idéologique, sécuritaire, moral et culturel» (2001: 10). Par ailleurs, la transformation des jeunes en tant qu'acteurs sociaux encourage la création de nouvelles formes de légitimité et de nouveaux espaces d'expression individuelle ou collective (Diouf, 2003). Ainsi, dans un grand nombre de sociétés africaines, les multiples transformations politiques et économiques des deux dernières décennies du $\mathrm{xx}^{\mathrm{e}}$ siècle, parallèlement à l'érosion des engagements de l'État et de la famille, mènent à des formes d'action politique associatives et esthétiques novatrices en marge des codes traditionnels de conduite.

Une nouvelle capacité d'action et d'activisme 4 des jeunes s'observe, qui correspond à une mutation radicale des modalités de leur participation aux débats de société. La constitution de nouveaux espaces d'expression pour les jeunes, avec notamment la création de forums publics, tend à proposer une conception moins restrictive de la participation citoyenne. En cela, les jeunes sont des acteurs qui bousculent l'échiquier social en personnifiant «les terreurs du présent, les erreurs du passé, les prévisions du futur, les anciens espoirs et les nouveaux défis» (Comaroff et Comaroff, 2000a: 92) et sont des figures ambivalentes car ils constituent "menace et promesse à la fois» (Comaroff et Comaroff, 2000a: 96). Le terme "jeunes» renvoie ici à une catégorie sociale dont l'âge varie de la puberté biologique à la quarantaine, regroupant surtout des hommes ${ }^{5}$, individus célibataires souvent sans grandes ressources économiques. À la fin du $\mathrm{xx}^{\mathrm{e}}$ siècle, dans un contexte de crise économique et de déstabilisation politique, cette catégorie sociologique s'élargit, menant ainsi à une redéfinition des statuts et des rôles sociaux.

Nous utiliserons la notion de «citoyenneté culturelle» élaborée par Toby Miller (1998) dans le cadre de ses travaux sur la technologie et la réceptivité des médias de masse aux États-Unis, et ce afin de répondre à trois objectifs principaux: 1) mieux cerner les pourtours du nouvel activisme des jeunes musulmans; 2) mesurer les variations du processus de réaffirmation de l'identité religieuse dans les milieux étudiants et associatifs, ainsi que ses logiques dans un contexte de fort regain religieux en Afrique de l'Ouest francophone, à partir de la fin des années 1970 et plus encore dans les années 1980-1990 ; 3) appréhender la capacité des jeunes musulmans à innover dans leurs stratégies de contrôle de l'espace public. Pour cela, nous proposons une synthèse de données empiriques originales provenant d'enquêtes menées au Sénégal, au Burkina Faso, en Côte d'Ivoire et au Mali'.

4. Correspondant au terme agency utilisé dans la littérature anglophone.

5. Voir LeBlanc, 2006b et 1998, pour une discussion détaillée de la notion de «jeune» en Afrique de l'Ouest; Hamel, 1999; Last, 1992 et d'Almeida-Topor, 1992.

6. Les enquêtes ont été menées par Muriel Gomez-Perez au Sénégal (1992-2005) et au Burkina Faso (2005, 2006) et par Marie-Nathalie LeBlanc en Côte d'Ivoire (1992-1995, 1998 et 2000) et au Mali (2003). 
Cet article s'inscrit dans la lignée des recherches sur les dynamiques identitaires islamiques en Afrique de l'Ouest'. Nous délaisserons ici les élites des diverses structures nationales de pouvoir pour étudier, d'une part, les jeunes hommes ${ }^{8}$ ayant une scolarisation postsecondaire, provenant de milieux laïcs francophones et, d'autre part, les arabisants scolarisés dans des madâris ${ }^{9}$ franco-arabes en Afrique de l'Ouest, au Soudan, au Maghreb, en Égypte ou en Arabie Saoudite.

Notre choix de confronter nos données empiriques sur ces quatre pays en particulier s'explique par plusieurs raisons. L'islam y est démographiquement majoritaire, voire ultra majoritaire au Sénégal et au Mali. Tout en maintenant son caractère laïc, à quelques nuances près ${ }^{10}$, l'État cherche à s'insérer davantage dans le champ du religieux (Gomez-Perez, 2005a) de ces pays où émerge depuis deux décennies une nouvelle et profonde dynamique de l'islam, avec la multiplication des mosquées, des associations islamiques, des madâris et de la presse islamique ${ }^{11}$. Cette dynamique s'accélère et touche différentes classes socioprofessionnelles depuis les années 1990, à la faveur des processus de démocratisation qui entraînent une libéralisation de l'espace public, notamment avec la multiplication des média islamiques. Cette libéralisation a, pour sa part, encouragé la transformation des relations entre aînés et jeunes musulmans (Gomez-Perez, LeBlanc, et Savadogo, 2007) et les relations sociales dans les quartiers urbains (Gomez-Perez, 2008).

7. Voir, entre autres, Alidou, 2005; Savadogo 2006, 2005; Janson, 2007; Soares, 2005; Miran, 2006; Schulz, 2006, 2003; Ilhe, 2003; Kane, 2003; Augis, 2002; Masquelier, 2001; Loimeier, 1997; Brenner, 1993a; Umar, 1988. Voir aussi Gomez-Perez, 2008, 2005a et b, 1999, 1998, 1994 et LeBlanc, 2008, 2007, 2006a et b, 2005, 2000, 1999.

8. Voir entre autres les travaux de Augis, 2005, 2002; Janson, 2007 et Alidou, 2005, ainsi que ceux de LeBlanc sur la Côte d'Ivoire, 2008, 2007, 2000. Ces écrits soulignent le fait que les motivations et la participation des jeunes femmes et des jeunes hommes à la revitalisation de l'islam diffère significativement. C'est pourquoi, à moins que cela ne soit spécifié, l'expression «jeunes musulmans» désigne les jeunes hommes.

9. Pluriel de madrasa, établissement d'enseignement supérieur islamique.

10. Au Sénégal, des associations islamiques se sont opposées à la laïcité de l'État dès la fin des années 1970 (voir notamment Gomez-Perez, 1997 et 1994; Fall, 1985 et 1983; Magassouba, 1985; Coulon, 1984 et 1983) en raison de relations étroites entre les confréries et la politique depuis la colonisation (voir écrits de Cruise O’Brien et de Coulon). Malgré tout, s'instaure une collusion de plus en plus grande entre État et islam qui conduit à parler de «laïcité compréhensive», de «mouridification» de l'État sous Abdoulaye Wade. Au Mali, la laïcité est à la fois contestée, maintenue et revendiquée (Soares, 2005; Brenner, 1993b). Au Burkina Faso, l'association Communauté Musulmane du Burkina Faso (CMBF) est encore aujourd'hui rattachée à l'État (Oumarou Kanazoé est président et argentier de l'association, principal entrepreneur de travaux publics du pays et intermédiaire entre État et musulmans dans des dossiers délicats (voir Gomez-Perez, 2008; Otayek, 1996a et 1984; Kouanda, 1989). En Côte d'Ivoire, face à la restructuration de la communauté musulmane autour du Conseil National Islamique (CNI), le Parti Démocratique de la Côte d'Ivoire (PDCI) au pouvoir jusqu'en 1993 a maintenu une position d'ouverture dans le contexte de la laïcité. Par ailleurs, la négociation entre le CNI et le PDCI concernant l'inclusion des madâris dans le curriculum scolaire national et la reconnaissance des fêtes religieuses musulmanes en tant que journées de congé national attestent de ce positionnement idéologique.

11. Nous renvoyons aux travaux suivants: mosquées, Gomez-Perez, 2008; Kounda, 1996; associations islamiques, Miran, 2006; Augis, 2002; LeBlanc, 1998; Gomez-Perez, 1997, 1994; Diaw, 1992; madâris, Brenner, 2001; Gomez-Perez, 1999, 1997; Diawara, 1996; Cissé, 1990 ; presse islamique, Schulz, 2006, 2003; Soares, 2005; Davis, 2002. 
Au-delà de ces similitudes, on ne retrouve pas dans les quatre pays la même chronologie ni la même dynamique de ferveur religieuse. Le Sénégal initie le mouvement avec la création dès 1978 de la Jamaatou Ibadou Rahmane (JIR) ${ }^{12}$. Dans les trois autres pays, il faut attendre les années 1990, marquées par une démocratisation et un pluralisme politique accru, pour observer tant une irruption des jeunes dans l'espace public qu'une dynamisation de l'islam. Ajoutons qu'en Côte d'Ivoire et au Burkina Faso, l'islam se voit marginalisé dans l'espace public (Miran, 2006; Savadogo, 2005; Gary-Tounkara, 2005; LeBlanc, 1998) ${ }^{13}$, où il doit composer avec les chrétiens (en position hégémonique) et les pentecôtistes récemment devenus plus influents (LeBlanc, 2003; Otayek, 1997) ${ }^{14}$. Avec la mort du président Houphouët-Boigny en décembre 1993 puis la course à sa succession, l'État ivoirien s'est progressivement replié sur un concept de citoyenneté ancrée dans un principe d'autochtonie, excluant ainsi la possibilité d'accommodation religieuse et culturelle pour les musulmans au sein des institutions étatiques (Marshall-Fratani, 2006). Au Burkina Faso, l'Islam ne parvient ni à «convertir en influence politique sa prédominance démographique» (Otayek et Diallo, 1998: 33), ni à utiliser son influence économique accrue depuis la politique du général Lamizana ${ }^{15}$.

De surcroît, dans ces quatre pays, les jeunes sont souvent maintenus en marge des sphères économique et politique, en raison de dynamiques historiques nationales ou de diverses politiques néolibérales. Toutefois, comme nous le verrons dans un premier temps, des processus hybrides s'articulent où l'individuation et la fidélité à la communauté religieuse cohabitent. Nous montrerons, dans un second temps, que la nouvelle capacité d'action et d'activisme des jeunes musulmans conduit à mettre en ouvre de nouvelles modalités de participation à la société et à remoraliser l'espace public. Dans les deux cas, les stratégies et les modalités d'expression adoptées par les jeunes musulmans se rapportent autant à la reconnaissance d'individualités qu'à la recomposition de communautés musulmanes locales.

\section{Mise en ceuvre d'Une nouvelle citoyen neté Par L'isLam dans L'espace PUBLIC}

Les quatre pays à l'étude subissent une grave crise économique depuis les années 1980 avec notamment la chute sur les marchés du prix du cacao et du café en Côte d'Ivoire,

12. Issue d'une scission de l'Union Culturelle Musulmane fondée à Dakar en 1953 (Gomez-Perez, 2005b, 1997).

13. Au Burkina Faso, après un partenariat important entre islam et État sous le régime du général Lamizana entre 1966 et 1980, la marginalisation de l'islam débute avec la révolution du 4 août 1983.

14. Dans le cas du Burkina Faso, en décembre 1998, le gouvernement, conscient de la popularité des Assemblées de Dieu, leur avait demandé de présider la Commission électorale nationale indépendante à l'occasion des élections présidentielles (Laurent, 2003: 250 et suivantes). En Côte d'Ivoire, la présidence de Laurent Gbagbo depuis 2000 marque l'insertion des néo-protestants dans le débat politique sous l'influence de son épouse Simone Ehivet Gbagbo, très proche des évangélistes états-uniens (les Églises évangéliques des Assemblées de Dieu).

15. Le général Lamizana avait permis aux musulmans de participer aux logiques du marché et de profiter des facilités financières instituées par l’État (voir notamment Labazée, 1988). 
et de l'arachide au Sénégal. Jusqu'à maintenant, les États peinent à contrôler cette crise et multiplient les mesures de libéralisation économique qui se sont dramatiquement accélérées à la suite de la dévaluation du franc CFA en 1994. Malgré l'euphorie qui suit certaines ruptures politiques — telles que le renversement du dictateur Moussa Traoré et l'élection de Alpha Oumar Konaré en avril 1992 au Mali, la défaite électorale d'Abdou Diouf et l'élection d'Abdoulaye Wade au Sénégal en 2000, ou encore les premières élections multipartistes en 1990 en Côte d'Ivoire —, la crise économique perdure et les différences sociales s'accentuent. Ainsi, les désillusions deviennent plus amères et laissent craindre des actes de violence.

Les jeunes sont les premières victimes de cette dégradation économique, et la violence devient la seule solution pour nombre d'entre eux, qu'ils soient marginalisés ou scolarisés. Au Sénégal, les jeunes participent à des émeutes urbaines, en février et en mars 1988 puis en février 1993, à la suite de la reconduction contestée du président de la République Abdou Diouf (Diop et Diouf, 1990: 285 et suivantes). En Côte d'Ivoire, ils se manifestent dans l'espace public à travers de violentes contestations initiées en milieu scolaire et universitaire contre le Parti Démocratique de Côte d'Ivoire (Konaté, 2003), et visant la réforme de la démocratie à parti unique ${ }^{16}$. Fin 1996, au Burkina Faso, les étudiants de l'université de Ouagadougou font grève pendant trois mois afin de dénoncer les conditions de travail et un régime de plus en plus autoritaire et corrompu depuis l'assassinat de Sankara, dix ans auparavant (Wise, 1998). Cette défiance vis-à-vis de l'État s'amplifie après l'assassinat du journaliste Norbert Zongo en décembre 1998, date à laquelle la société civile regroupe en un même collectif diverses organisations démocratiques de masse et divers partis politiques pour lutter contre l'impunité et demander justice (Hagberg, 2002; Loada, 1999). Finalement, au Mali, les jeunes participent aussi activement au processus de démocratisation en jouant un rôle central dans le renversement du dictateur Moussa Traoré en 1991 (Brenner, 2003; Cissé, 1985).

L'exclusion politique et économique des jeunes au cours des années 1980 les a conduit à se créer des espaces multiples, parallèles à ceux créés par l'État, au sein desquels le religieux devient objet de revendication, de négociation et de pouvoir. De fait, le religieux se transforme pour les jeunes en une arène où se jouent leur consentement et leur résistance aux modèles de socialisation existants. Afin de cerner les nouvelles dynamiques de l'action politique des jeunes Africains marginalisés, Jean et John Comaroff (200ob: 308) avancent la notion de "contre-nation»: « une citoyenneté virtuelle avec sa propre économie naissante, ses propres espaces de production et de récréation, ses propres modalités politiques à l'aide desquelles elle adresse les conditions économiques et politiques qui la déterminent» [traduction].

Toutefois, pour mieux circonscrire les pourtours de l'activisme des jeunes musulmans, il nous paraît plus pertinent de reprendre la notion de «citoyenneté culturelle» proposée par Toby Miller (1998). Cette notion désigne les pratiques des

16. CE sont ces mêmes jeunes, membres de la Fédération des Élèves et Étudiants de la Côte d'Ivoire (FESCI) qui ont nourri la guerre civile de 2002 (notamment Guillaume Soro et les Forces nouvelles au nord, et Blé Goudé et les jeunes patriotes d'Abidjan) (Konaté, 2003). 
spectateurs qui reconfigurent l'espace public et les enjeux de la citoyenneté dans les sociétés démocratiques. Chez Miller, la citoyenneté ne se limite pas au statut légal du citoyen et à la relation exclusive entre un État, une population donnée et un territoire spécifique. Tandis que la citoyenneté nationale correspond à un statut juridique (définie en termes généraux de droit de vote, droit de se porter candidat aux élections, droit de détenir un passeport), la citoyenneté culturelle se revendique au-delà des limites juridiques de l'État. Elle évoque un ensemble de pratiques et de droits (droit à l'expression, à l'auto-détermination identitaire, à la diversité, etc.) à même de suggérer qu'une société donnée ne se limite pas à un espace politique et économique restreint. Tout comme le concept de contre-nation, le concept de citoyenneté culturelle ne se borne pas à articuler identité et territoire; il évoque une dimension déterritorialisée dans la mesure où cette citoyenneté crée des espaces et des actions qui encouragent le débat public, favorisent l'échange d'idées et permettent à des groupes d'individus souvent marginalisés, sinon exclus, d'exprimer leurs identités et de revendiquer leurs propres projets moraux. Cependant, les pratiques associées à la citoyenneté culturelle, contrairement aux pratiques associées à la contre-nation, ne s'inscrivent pas nécessairement en porte-à-faux ni en opposition à l'État ou à la nation.

De fait, cette notion de citoyenneté culturelle concorde avec les observations réalisées sur nos terrains d'enquête respectifs: les jeunes musulmans investissent l'espace public sans pour autant ignorer l'État. Ils veulent en réalité instaurer coûte que coûte un dialogue avec l'État afin que celui-ci ne les ignore pas et tienne compte minimalement de leurs aspirations et de leurs projets moraux de société. En revanche, la violence contre l'État survient au moment où les jeunes musulmans prennent conscience que le dialogue est biaisé ou inexistant, dans des contextes de tensions politiques imputables à des États autoritaires, dirigistes et dominants ${ }^{17}$, ou à des démocraties en trompe-l'œil ${ }^{18}$. C'est le cas, par exemple, lors de l'annonce diffusée à la télévision du plan de déguerpissement de la population du quartier Zangouettin à Ougadougou en 2001-2002, décidé sans concertation. Cette action provoque la colère des habitants et notamment des jeunes:

Après l'annonce du projet le 14 février 2001 au journal télévisé de 20 heures, il y eut spontanément une grande émeute dans le quartier, tous les jeunes du quartier et des quartiers environnants étaient dehors. Environ 50 vieux sont passés chez moi afin de m'envoyer calmer les jeunes dans la rue. C'est alors que je demandais à mon chauffeur de m'amener sur l'avenue Yennenga (c'est là que se trouvaient les grévistes). La foule s'étendait de la maternité Yennenga au Lido bar. J’ai donc marché tout au long de l'avenue pour implorer aux jeunes le calme, grâce à Dieu j'ai réussi à les calmer, juste avant l'arrivée des forces de l'ordre (Entretien avec imâm Bangré, Ouagadougou, août 2006).

17. Pour le Burkina Faso, par exemple, voir notamment le numéro 101 (mars-avril 2006) de Politique africaine intitulé «Retour au Burkina Faso: l'alternance impossible»; Otayek et alii, 1996b; Otayek, 1989.

18. Voir le numéro 45 (mars 1992) de Politique africaine intitulé «Sénégal. La démocratie à l'épreuve» et le numéro 96 de Politique africaine (décembre 2004) intitulé «Sénégal 2000-2004, l'alternance et ses contradictions». 
Alors que Nancy Dolby (2006) avance que la culture populaire en Afrique est devenue un espace privilégié d'expression pour les jeunes en situation de marginalisation, nous souhaitons pour notre part étendre la notion de citoyenneté culturelle au champ du religieux, afin de mettre en lumière le rôle joué par la religion chez les jeunes dans l'Afrique postcoloniale et néolibérale.

Dans les années 1990, il s'agit de plus en plus pour les jeunes de vivre quotidiennement et activement leur foi afin qu'elle se diffuse largement. L'islam revendiqué par les jeunes, hommes et femmes, consiste en une modification radicale du quotidien de chacun afin de spécifier son identité islamique. La plupart des témoignages collectés montrent qu'il s'agit d'abord de manifester leur différence dans leurs rapports à l'Autre et dans leur approche de l'islam. C'est ainsi qu'ils mettent l'accent sur l'esthétique, l'hygiène corporelle, le port de la barbe à une certaine longueur à l'instar du prophète, le port du voile pour les femmes, le salut en langue arabe, le fait de ne pas serrer la main à une femme (autre que l'épouse) et de faire le Hajj beaucoup plus jeune ${ }^{19}$. Il s'agit aussi de modifier les fréquentations, les lieux et les modalités de socialisation, pour mieux «(re)nouer des liens, (...) susciter de nouvelles amitiés» (Hervieu-Léger, 1990: 146). Ainsi émerge un nouvel homo islamicus pour lequel la foi est à lier tant à l'expérience individuelle qu'à l'expérience collective, afin de mieux «se retrouver "entre soi"» (Hervieu-Léger, 1990: 148), dans une atmosphère complice et chargée d'émotion qui invite au renouveau de l'engagement confessionnel.

L'ensemble des discours que nous avons recueillis auprès de jeunes hommes met en exergue la figure du «bon musulman ${ }^{20}$ apparue dans les années 1980 et qui se renforce dans les années 1990. Le «bon» musulman a une foi sans faille, met l'accent sur la pratique de l'islam, prie et sait comment prier, se comporte au quotidien selon les préceptes du Coran et de la Sunna, suit la voie du prophète, est bien guidé, et a une conduite de vie irréprochable et exemplaire.

En cela, les jeunes musulmans militants se constituent comme des sujets moraux, prônant une conduite de vie et une remoralisation de l'individu et de la société dans son ensemble (Marshall-Fratani et Péclard, 2002). Le «bon» musulman joue ainsi le rôle du missionnaire pour l'expansion et l'enracinement de la dimension éthique de l'islam dans la société, ce qui lui confère un statut politique. Au cours des années 1990, à Bouaké par exemple, il est fréquent de croiser ces «vigiles islamisants», des jeunes qui tentent, dans le cadre de la vie quotidienne et à l'échelle des quartiers, de convaincre les autres jeunes musulmans de conformer leurs pratiques aux préceptes de l'islam, de réaliser leur foi et de se joindre aux activités des associations islamiques de quartier. À Bamako et à Ségou (Mali), les jeunes prosélytes se servent régulièrement de l'enregistrement de prônes sur cassettes audio pour exhorter à la réforme de la pratique religieuse et à la ferveur de la foi (Davis, 2002). À Djenné, les jeunes profitent

19. Voir LeBlanc, 2006b et 2005, pour une description des changements de ces marqueurs culturels en Côte d'Ivoire; voir aussi Soares, 2005 pour le Mali et Augis, 2005 pour le Sénégal.

20. Les jeunes femmes ont aussi un discours centré sur la question de la «bonne musulmane», mais ce comportement se définit différemment (voir Alidou, 2008; Masquelier, 2008; LeBlanc, 2007). 
du marché hebdomadaire du lundi pour acquérir des cassettes de prônes proférés par des prédicateurs connus (du Mali et d'ailleurs en Afrique de l'Ouest) qu'ils écoutent et commentent en groupe. Les étalages de marchés autour des mosquées, où se vendent ces enregistrements, sont aussi des espaces privilégiés de prosélytisme ${ }^{21}$.

De surcroît, beaucoup d'articles tirés des journaux islamiques étudiants portent sur les pratiques rituelles et sur la morale ou la moralité des comportements. Voici des extraits de An-Nasr, organe de presse de l'AEEMB (Burkina Faso):

La foi devient un facteur d'épanouissement individuel et collectif. Parce que le musulman est porteur d'un projet de société, la société dans laquelle il vit, il doit tirer profit de sa foi. Un bon musulman est donc un bon citoyen [...]. Les actes cultuels ont pour finalité d'éduquer le musulman et de faire de lui un homme de bien. [... ] Notre identité musulmane nous exhorte à voir un engagement citoyen au nom de Dieu et pour les hommes. Cette identité s'éduque pour transmettre la lumière, c'est agir pour construire la cité (Tiemtoré, Imam T., «Foi et citoyenneté», An-Nasr, $1^{\text {er }}$ février 2004: 1-3).

Les effets de la prière ne se limitent point à la sphère individuelle du fidèle. Ils passent cette limitation pour conquérir l'environnement dans lequel évolue la vie humaine (Sibiri, A.H., «La prière, une adoration et une éducation », An-Nasr, 6 février 2004: 2).

L'importance accordée à la prière dans les années 1990 rappelle ce qui pouvait être observé sur le terrain dans les années 1970-1980 lorsque plusieurs communautés musulmanes locales (notamment en Côte d'Ivoire, au Burkina Faso et au Mali) ont vu resurgir le débat entre ceux qui priaient les bras ballants et ceux qui priaient les bras croisés, assimilés aux Wahhabi par les colons français ${ }^{22}$. Mais l'enjeu a changé de nature en l'espace de vingt ans. Dans les années 1970, l'enjeu pour la nouvelle génération de musulmans, en tant que nouveaux militants actifs pour la cause de l'islam, était de conquérir un espace de reconnaissance et d'autonomie ${ }^{23}$ face aux autres tendances de l'islam (Gomez-Perez, 2005a et b). À partir des années 1990, la reconnaissance des jeunes militants musulmans n'étant plus si âprement discutée, il s'agit davantage d'exporter une vision de l'islam afin de «réislamiser» toutes les composantes de la société. Deux stratégies parallèles et complémentaires sont utilisées à cet effet. D'une part, il s'agit de questionner chaque personne sur son rapport à Dieu pour la ramener sur le bon chemin, en mettant ainsi l'accent sur la relation personnelle que tout croyant entretient avec Dieu ${ }^{24}$, et donc sur la centralité de l'expérience de la foi et de la transformation religieuse individuelle, ou conversion. D'autre part, les jeunes, en tentant d'investir avec force tout l'espace public, encouragent de plus en plus le militantisme religieux qui «offre la possibilité de se doter d'une image positive de soi, stable et valorisée» (Tozy, 1995: 117). Il ne s'agit donc pas d'un processus d'individualisation en émergence, dans la mesure où l'expérience de croyant n'est pas

21. Ceci a été observé dans les quatre pays.

22. Ce débat remonte aux années 1950, notamment au Mali. Voir Brenner, 2001 et Kaba, 1974.

23. Voir Miran, 1998: 18 et suiv. Voir aussi les luttes engagées contre la CMBF par le Mouvement Sunnite pour implanter des mosquées à Ouagadougou (Gomez-Perez, 2008; Kouanda, 1996).

24. Ceci n'est pas propre à l'islam politique mais aussi à l'islam confrérique, voir Audrain, 2004. 
seulement personnelle, mais aussi collective et communautaire (individuation). Car, sur la durée, cette image positive de soi complémente le sentiment de solidarité et de fraternité qui se développe au sein de diverses structures communautaires telles que les associations islamiques. L'image positive de soi doit servir aux autres, dans la mesure où tout un chacun devient un exemple à suivre et prend la fonction de guide, par sa fidélité aux normes du mouvement auquel le militant adhère. Il n'y a pas ici affranchissement vis-à-vis de la communauté. Chaque fidèle devient un militant actif pour la cause de l'islam.

Alassane, un jeune Ivoirien musulman, exprime la nuance entre le processus d'individuation et celui d'individualisation:

Ici [à l'association], je me sens utile. Nous avons des réunions hebdomadaires; nous organisons des prêches; nous lavons des mosquées; nous éduquons les autres jeunes du quartier. Avant, je traînais dans le quartier à rien faire. Maintenant, je vis ma foi. En plus d'être devenu un bon musulman, de m'être retrouvé, je suis un exemple pour mes frères plus jeunes. Et je contribue à l'avancement de l'islam. Que je sois un bon musulman, c'est toute la umma qui en profite, pas seulement moi (Bouaké, février 1994, traduit du dioula).

L'expérience de Arkhman, un Malien arabisant de Ségou, fait état de cette tension entre l'expérience individuelle de la foi et la fidélité active à la communauté:

Avant de changer ma pratique, je traînais dans les grin du quartier. On buvait du thé, on jouait aux cartes, on écoutait la radio, mais sans but, sans rien de précis à faire. Depuis que j'ai trouvé ma voie et que je me suis réinvesti dans ma religion, j'ai orienté mes actions. Je ne passe pas mes journées à flâner. Je prie beaucoup et je développe ma foi, mon savoir. Je lis, je discute avec les plus instruits, les plus vieux aussi. Je participe aussi aux activités de l'association. En dehors de nos actions dans la foi, nous engageons aussi des actions auprès de la communauté. L'association organise des corvées publiques. L'autre jour, nous avons nettoyé les rues du quartier (Ségou, novembre 2003, traduit du bambara).

La spécificité de l'affirmation religieuse de ces jeunes tient au fait qu'ils revendiquent une adhésion à l'islam à titre individuel pour mieux former une nouvelle communauté islamique, ce que plusieurs jeunes, comme Alassane, assimilent à la umma (communauté des croyants en islam), et que Farhad Khosrokhavar, renvoyant à l'islam des jeunes dans les banlieues françaises, nomme "une forme néo-communautaire», « une nouvelle collectivité dans la foi», «sur la base d'une adhésion volontaire» (1997: 30-31). Ces jeunes se situent par rapport «à une communauté islamique à construire et non pas donnée d'avance» (Khosrokhavar, 1997:58). Or l'image positive de soi en tant que croyant est la condition première pour donner une cohésion au groupe et pour mettre en œuvre sa participation au projet moral de la société. Ainsi, à travers cette image positive de soi et cet activisme, les jeunes s'approprient un droit à l'expression et un droit à l'auto-détermination identitaire. Au Burkina Faso et en Côte d'Ivoire, pays marqués par la diversité confessionnelle, les jeunes musulmans revendiquent aussi le respect de leur différence. 


\section{II - Remoralisation de L'espace public par les Jeunes musulmans}

À la lumière des modalités de mise en œuvre de la citoyenneté culturelle par les jeunes musulmans, il s'agit maintenant d'appréhender les conditions dans lesquelles ces derniers investissent l'espace public. La remoralisation de l'espace public par les jeunes musulmans conditionne l'émergence d'une «sphère publique post-séculaire», pour reprendre l'expression popularisée par Craig Calhoun (2007). Dans la mesure où les discours et les pratiques religieuses investissent l'espace public, il nous paraît essentiel d'analyser le rôle substantiel joué par la religion dans les débats publics et dans l'élaboration de divers modèles de sociabilitées. Dans cette perspective, l'espace public ne se conçoit pas seulement en tant qu'espace discursif où des messages politiques, sociaux et religieux sont échangés, mais aussi en tant qu'espace où l'activisme des jeunes met en scène les pratiques entourant l'imân (foi) et la da $a^{c} w a$ (prosélytisme). «Être musulman», c'est avant tout être actif tant dans sa foi que dans sa pratique du terrain par le prosélytisme.

Divers exemples de sermons publiés dans des médias islamiques, écrits par de jeunes musulmans, illustrent l'articulation entre foi et prosélytisme:

Mes frères et sœurs en la foi, même le monde islamique vit une crise d'ordre spirituel en ce sens que le Tawhîd, l'Unicité de Dieu, n'y est pas effectif. Il a besoin de se ressourcer d'abord pour pouvoir prétendre à la direction du monde. [...]. Cette crise que nous vivons ne doit point nous mener vers le désespoir, le défaitisme ou le fatalisme. Au contraire, elle doit nous pousser à accomplir un sursaut et à opérer un changement profond. [...]. Nous devons donc amorcer un retour vers l'islam, mais un retour soutenu par la science et la recherche constante du savoir. Pour cela, nous devons être des musulmans déterminés, engagés et responsables. Sachons que l'islam est une foi, il n'est pas héréditaire. Être issu d'une famille musulmane ou être natif d'un pays musulman ne suffit pas pour faire de l'individu un musulman. Ce dernier a le devoir de faire l'effort personnel pour mériter son appellation en s'engageant résolument dans la voie islamique (L'étudiant musulman, organe de presse de l'AEMUD, $\mathrm{n}^{\circ}$ 24, novembre-décembre 1996: 4-5).

Chers frères et sœurs, le jeûne du mois de ramadan nous rappelle notre appartenance à une communauté de foi et de devoirs. Nos associations, mouvements et autres fédérations ne sont que des moyens de faire la promotion de l'islam et non une finalité. Aussi les responsables se doivent-ils d'être des guides par des valeurs telles la modestie d'Abou Bakr, la rigueur d'Omar, la générosité d'Ousmane et le courage d'Ali, valeureux élève soumis à l'enseignement du prophète Mohammad. [...]. Nous avons l'obligation au nom de Dieu d'être dirigeants et dirigés, des hommes utiles à leur communauté. [...] Les plus martyrisés, opprimés, troqués, chassés et tués sont aujourd'hui des musulmans. Mais la religion qui progresse le plus et en dépit de tout est l'islam. [...] En Europe, les jeunes remplissent les mosquées tandis que les vieillards remplissent les églises ( $\mathrm{An}$-Nasr, $\mathrm{n}^{\circ} 44$, novembre 2004: 2-3).

25. Dorothea Schulz, 2006 et Benjamin Soares, 2005 dans le cas du Mali, ainsi que Adeline Masquelier, 2001 pour le Niger soulignent aussi la centralité de la religion dans les débats publics des années 1990. 
Par ailleurs, les jeunes musulmans ont pris des initiatives et multiplié les lieux d'expression pour rendre compte de leur engagement dans l'espace public ${ }^{26}$ et y affirmer leur identité islamique, concurrençant ainsi les lieux créés par les instances étatiques. Ce processus débute dans les années 1980 et s'accélère au cours des années 1990 lorsque les jeunes constatent leur exclusion des sphères politique et économique. Exclusion qui peut avoir pour cause la mainmise de l'État dirigiste (au Burkina Faso à partir de la révolution sankariste en 1983), la complicité ancienne entre l'islam confrérique et l'État (au Sénégal), la mise en place de mesures d'ajustement structurel reléguant les jeunes à la périphérie des activités économiques (dans les quatre pays étudiés) ou encore le caractère figé des débats autour de la citoyenneté (en Côte d'Ivoire).

C'est ainsi que les jeunes musulmans élargissent le cadre sociogéographique de leurs actions. Ils affichent leur identité islamique en investissant plus largement l'espace tant géographique, culturel que discursif, jusqu'à le saturer progressivement par le religieux (au Sénégal et au Mali) ou à y créer une forte compétition entre les différentes communautés religieuses (au Burkina Faso et en Côte d'Ivoire). Ainsi, au tournant du $\mathrm{xxI}^{\mathrm{e}}$ siècle, l'activisme des jeunes musulmans s'observe dans trois lieux privilégiés: les associations islamiques, nationales ou de quartier, les mosquées et les médias.

La transformation récente de la vie associative relève autant d'enjeux mondiaux que de dynamiques locales propres aux divers espaces nationaux discutés ici. En effet, elle s'inscrit dans un contexte de début de regain islamique au cours des années 1980, de «démocratisation» au début des années 1990, mais aussi d'exclusion en Côte d'Ivoire avec la mise en exergue de la notion d'ivoirité. C'est ainsi que de nouvelles associations islamiques voient le jour, dont le Conseil National Islamique (CNI) en Côte d'Ivoire autour duquel se restructure la communauté musulmane (LeBlanc, 2006b, 1998; Miran, 2006; Savadogo, 2005). Ce sont au premier chef les jeunes étudiants des milieux scolaires laïcs qui fondent des associations ${ }^{27}$. Les madâris aussi encouragent la création d'associations étudiantes, au même titre que certaines mosquées. Les associations islamiques de quartier se multiplient également de façon fulgurante dans les années 1990.

26. Les jeunes femmes sont aussi actives dans cet engagement. Par exemple, en Côte d'Ivoire, elles initient souvent la création d'associations de quartier regroupant de jeunes musulmans. Toutefois, bien qu'actives dans les associations de femmes musulmanes, ces dernières n'ont que très rarement ou même jamais un rôle public et central dans les mosquées étudiantes, dans les associations ou dans les madâris. Leur activisme transparaît, entre autres, dans les œuvres de charité ou plus récemment dans les ONG confessionnelles (LeBlanc, 2008).

27. Dont l'Association des Étudiants Musulmans de l'Université de Dakar (AEMUD), l'Association des Étudiants et Élèves Musulmans du Burkina (AEEMB) (Gomez-Perez, 2005a, 1997, 1994), l'Association des Étudiantes et Élèves Musulmans du Sénégal (AEEMS), l'Association des Étudiants et Élèves Musulmans de Côte d'Ivoire (AEEMCI), l'Association des Jeunes Musulmans de Côte d'Ivoire (AJMCI), l'Association islamique pour l'épanouissement et le développement, les Pieds Nus, l'Association de la solidarité islamique, ou encore la très populaire association Ancar Dine (Davis, 2002). 
De même, le rythme de construction des mosquées s'accélère considérablement depuis 15 ans. Ayant mis la $d a^{c} w a$ au centre de leur stratégie, les jeunes hommes luttent pour obtenir la construction de mosquées sur les campus universitaires, militent dans des associations pour la construction de mosquées dans tous les types de quartier sans exception (populaires comme aisés, résidentiels, périphériques comme centraux, non lotis ou encore dans des zones fragiles), dans le but de développer et structurer des réseaux sociaux et d'affirmer leur identité islamique (Gomez-Perez, 2008). Dans un contexte de forte concurrence entre les associations œuvrant pour la construction de mosquées du vendredi et pour l'enracinement de la $d a^{c} w a$, les jeunes sont de plus en plus invités par leurs aînés à participer à la diffusion du prône du vendredi, quelle que soit la nature doctrinale du mouvement islamique gérant le lieu (au Sénégal et au Burkina Faso) (Gomez-Perez, 2008; Gomez-Perez, LeBlanc et Savadogo, 2007). La vignette ci-dessous atteste des modalités d'appropriation physique et sociale de l'espace urbain par les jeunes musulmans:

Depuis 1993, l'Association des jeunes Musulmans de Air France 1 (un quartier de Bouaké) organise des prêches dans les lieux publics du quartier. En 1995, pour le Ramadan, l'association a organisé un prêche sur la place de la Paix, un énorme rond-point de $300 \mathrm{~m}^{2}$, au carrefour du Commerce (le quartier commercial), du grand marché et de deux quartiers d'habitation. L'association a loué des chaises qui sont alignées comme dans une salle de spectacle. Il y a plus de 200 spectateurs vêtus de blanc. La scène improvisée est dominée par une longue table nappée de blanc à laquelle sont assis cinq hommes devant des micros. Ce sont les prédicateurs qui livreront le sermon de la soirée. Il y a des haut-parleurs et des projecteurs lumineux de chaque côté de la table. Les cinq conférenciers comprennent le président de AMAR qui enseigne le français dans une école nationale, son conseiller en matière de religion qui enseigne dans une madrasa, le conférencier principal qui provient de la Mauritanie, un membre de la section régionale de la Ligue des prédicateurs de Côte d'Ivoire et le directeur de la principale madrasa de Air France (Bouaké, avril 1995).

Les jeunes transforment les enjeux du prosélytisme en usant de nouvelles méthodes et en s'engageant davantage à divulguer la religion, notamment à partir des années 1990. Il est frappant de voir de jeunes prêcheurs investir les ondes de la radio et de la télévision (Mali, Sénégal, Côte d'Ivoire). La diffusion de prônes sur cassettes audio initiée dans les années 1980 s'étend dans les années 1990, tout comme celle de pamphlets, d'autocollants, de calendriers, de banderoles, de vêtements ( $t$-shirts et foulards de prière), lesquels publicisent des prédicateurs (hyper)médiatisés. L'instrumentalisation des médias maliens par le prédicateur vedette Cherif Ousmane Madani Haïdara est à cet égard un exemple remarquable (Schulz, 2006; Soares, 2005; Davis, 2002) : les enregistrements de ses prônes circulent à travers toute l'Afrique de l'Ouest, principalement auprès des jeunes (Traoré, 2005). Cette diffusion contribue, comme le souligne très justement Olivier Roy, à «la circulation de savoirs religieux dans les réseaux non contrôlés » (1999: 23), sans pour autant remettre en cause d'autres systèmes de savoir plus conventionnels, tels que ceux dispensés dans les madâris. Soulignons toutefois quel'hypermédiatisation des prêches ne concerne pas uniquement 
les jeunes prédicateurs, mais aussi leurs aînés. Parallèlement à la notoriété ascendante de plusieurs imâm d'une autre génération ${ }^{28}$, de jeunes imâm sont reconnus comme des prédicateurs charismatiques par leur communauté pour plusieurs raisons: ils ont une bonne connaissance du Coran et de la Sunna; ils ont, pour certains, poursuivi des études islamiques à l'étranger; ils ont des talents d'orateur qui leur permettent de diffuser leur message et de rendre compte de l'actualité nationale ou internationale, et par là même d'attirer les fidèles ${ }^{29}$. Ainsi le prêcheur est avant tout un messager, voire un guide des esprits.

Par ailleurs, cette diffusion des prônes participe plus largement d'une médiatisation de toute action de nature religieuse à fort impact émotionnel. Par exemple, afin de capter l'attention des médias sur le sort des résidants contraints de quitter le quartier Zangouettin à Ouagadougou, les jeunes ont choisi de nommer leur mouvement local d'opposition Al Qaida, étant captivés par les questions internationales, voire fascinés par la dimension médiatique de certains mouvements islamiques à l'échelle planétaire: «Le mouvement tient son nom du jeune Peul qui donnait les informations à tout le quartier. On l'appelait Al Qaida, et l'année en question, Al Qaida était à la une de la presse. Pour donc faire important et effrayant, nous avons choisi de nous faire appeler Al Qaida» (Ouagadougou, septembre 2006).

Outre les associations et les mosquées, les jeunes investissent d'autres espaces pour exprimer leur activisme, comme la rue, les stades ou les grands hôtels, des lieux qui ne sont pas à proprement parler religieux, mais qui par leur visibilité permettent d'attirer toutes les couches de la société aux débats islamiques. Par l'utilisation de tels espaces il s'agit de mettre en scène la foi et de décloisonner l'islam, tant sur le plan géographique qu'idéologique ou social. Investir un stade, c'est non seulement investir un lieu populaire où sont organisés de grands rassemblements, mais c'est aussi prendre possession d'un lieu originellement laïc et ludique afin de l'islamiser. Ce phénomène est très marqué au Mali et en Côte d'Ivoire. Le CNI organise depuis 1993 à la fin du Ramadan une nuit de prière dans divers stades, notamment au stade de Bouaké et au Grand stade d'Abidjan. Investir un grand hôtel, c'est sensibiliser des milieux nouvellement convertis ou à convertir (cadres, intellectuels, industriels, politiques, étrangers) dans un lieu généralement luxueux, afin que l'islam ne soit plus associé dans l'imaginaire collectif à une religion des seuls déclassés ou à une religion marginale dans la sphère publique et politique, comme c'est le cas au Burkina Faso ou en Côte d'Ivoire.

28. Tels que Mohamed Seyni Gorgui Guèye, l'imâm fondateur de la mosquée dite tombée du ciel à Dakar, de Mbaye Niang, imâm de la mosquée inachevée de Yoff à Dakar, de El Hadj Aboubacar Sana, imâm de la grande mosquée gérée par la CMBF à Ouagadougou, de feu El Hadj Ouedraogo Sayouba de la grande mosquée du vendredi gérée par le Mouvement Sunnite dans le quartier Zangouettin à Ouagadougou, ou de l'imâm Saïdou Bangré, de la confrérie tidiane, connu pour avoir dirigé le mouvement d'opposition face au déguerpissement de la population du quartier Zangouettin dans le cadre du projet ZACA.

29. Par exemple, l'imâm Kanté de l'AEMUD, l'imâm Niang à la mosquée du Point E à Dakar, ou encore les prédicateurs formés par la LIPCI à Abidjan, ainsi que certains imâm abidjanais formés à l'étranger, dont certains ont vécu en Europe. 
Ce changement de stratégie s'explique de trois manières. D’abord par le profil des jeunes prédicateurs. Les uns, après avoir étudié dans des universités arabo-musulmanes où ils ont été sensibilisés aux techniques de prosélytisme, décident d'employer ces mêmes stratégies de retour au pays. Les autres, francophones, veulent investir les lieux dits occidentalisés. Plutôt que de rejeter les éléments structurants de la vie urbaine ou de la mondialisation, ils souhaitent les convertir aux règles fondamentales de l'islam. Ce changement de stratégie s'explique aussi par la volonté des jeunes leaders islamiques de contrer le prosélytisme des mouvements pentecôtistes (notamment en Côte d'Ivoire et au Burkina Faso) et de certains mouvements confrériques tels que les Moustarchidines au Sénégal, dans lesquels il est recommandé aux taalibé (disciples) de Moustapha Sy de propager les enseignements du mouvement à l'ensemble de la société en montrant le bon exemple ou en éduquant les cadets (Samson, 2005). Enfin, le fait de mettre en scène sa foi représente aussi un acte de résistance face à une marginalisation politique des musulmans (Burkina Faso) ou face à une menace (Côte d'Ivoire) $)^{30}$.

Ajoutons que l'organisation de caravanes de prêche ${ }^{31}$, le scoutisme, les colonies de vacances (Gomez-Perez, 2005b), les actions caritatives sont des stratégies de prosélytisme qui se rapprochent de celles de l'Église catholique et des mouvements pentecôtistes $^{32}$. Le Sénégal apparaît ici comme un pionnier dans le domaine caritatif, avec la création de l'association Jamra en 1985, transformée en ONG en 1988. Ses actions portent sur l'enfance déshéritée, la lutte contre la drogue, la lutte contre le sida ou encore en 1989 l'aide aux réfugiés de Mauritanie. Ce type d'action a aussi pris de l'ampleur en Côte d'Ivoire avec la création d'un certain nombre d'ONG ${ }^{33}$.

Ainsi, ces diverses manifestations de foi montrent à quel point les jeunes s'inscrivent totalement dans les logiques de la modernité: le prosélytisme répond aux lois du marché religieux ambiant tout en réinventant les termes de cette modernité (Comaroff et Comaroff, 1999: 3). Ces jeunes musulmans s'adaptent aux règles du marché religieux, font imploser les frontières entre espace public (rue, ville, espace médiatique) et espace privé (cour de la maison) pour les rendre plus fluides, plus poreuses. En outre, leur stratégie, plus ou moins consciente suivant les cas, consiste non seulement à contourner un État trop hégémonique et totalisant (Burkina Faso et Côte d'Ivoire), mais aussi et surtout à contrecarrer, voire à concurrencer l'État sur son propre territoire en profitant de chaque opportunité offerte par la démocratisation pour prendre la parole.

30. Cette stratégie n'est pas propre à l'Afrique de l'Ouest, elle s'observe aussi en Afrique australe, voir notamment Sadouni, 2002: 101-109.

31. Cette stratégie de prosélytisme en Côte d'Ivoire a aussi été décrite par Marie Miran, 2000: 151-154.

32. Ousmane Kane pour le Nigéria tout comme Abdoulaye Sounaye et Souley Hassane pour le Niger observent les mêmes stratégies.

33. Foi et Solidarité, Secours Médical Islamique (Miran, 2000), ID-Pan (Intelligentsia de la Diaspora Panafricaine) établie après la crise de 2002, Fondation Djigui, rattachée à des ONG américaines (LeBlanc, 2006b). 


\section{Conclusion}

Les jeunes musulmans d'Afrique de l'Ouest se réclament ainsi d'une forme de citoyenneté culturelle en investissant divers espaces qui ne leur étaient pas réservés, soit en raison du poids incontournable des aînés dans les institutions traditionnelles, soit en raison de la politique hégémonique de l'État. Ils ont pour projet de donner un cadre moral à la société, en opposition avec les modèles privilégiés par la modernité dite occidentale. De fait, leur version de l'islam propose une troisième alternative d'action populaire face au couple modernité/tradition ${ }^{34}$. L'islam devient ainsi une structure morale essentielle de la modernité. Cette charpente morale repose sur une nouvelle façon de vivre l'islam, et non simplement sur l'instrumentalisation du religieux à des fins sociales et politiques ou de la religion comme espace de revendication. La remoralisation de l'espace public par l'islam s'articule autour d'un nouvel homo islamicus.

Par ailleurs, il apparaît très clairement que l'émergence d'une individualisation de nature islamique n'est pas systématique dans l'itinéraire des jeunes musulmans. Comme nous l'avons souligné, à partir des années 1990, l'activisme des jeunes repose sur une juxtaposition de stratégies mêlant le collectif et l'individuel; les frontières entre les stratégies prosélytes issues de différentes tendances de l'islam sont plus poreuses; des identités islamiques polymorphes émergent. Certains jeunes gagnent en légitimité religieuse, mais aussi en autonomie, grâce notamment à la bonne visibilité et à la bonne réception de leurs prônes ou à la reconnaissance de leurs savoirs religieux. D'autres (en particulier des jeunes de famille confrérique, qui publicisent la fondation de mosquées) utilisent «leur capital social communautaire à des fins personnelles» (Marie, 1997: 9). Ces deux catégories de jeunes participent du processus d'individualisation auquel correspondent des pratiques de vedettariat ou d'instrumentalisation du religieux. Cependant, la grande majorité des jeunes musulmans adhèrent à des associations religieuses, à des ONG islamiques, organisent des caravanes de $d a^{c} w a$ tout en restant dans l'anonymat, car l'objectif est d'aller vers l'Autre pour convertir ou réislamiser en masse et avec rigueur. L'ultime but est d'utiliser son expérience personnelle pour servir la communauté islamique dans son ensemble. Cette majorité regroupe des sujets hybrides ni totalement individualisés et autonomes, ni totalement communautaires ${ }^{35}$. Ils visent à retrouver la cohésion propre à la $u m m a$ des premiers temps de l'islam, selon la logique de l'association ou de l'organisation dans laquelle ils militent activement et volontairement. En cela, l'individualisation

34. Voir LeBlanc, 1998 pour une analyse de la relation ambiguë à la modernité chez les jeunes arabisants de Côte d'Ivoire. Marie Miran propose pour sa part une analyse de la modernisation de l'islam des années 1990 en Côte d'Ivoire.

35. Alain Marie définit ainsi le sujet communautaire: «C'est toujours par référence à une logique communautaire dominante que des individus, plus fortement individués que d'autres, n'en sont pas moins assignés à des places sociales prédéterminées à l'intérieur d'un système de «jeux de rôles» préexistant à la manifestation de leur individualité et leur imposant des conduites stéréotypées, assujetties aux normes communautaires et à leur reproduction...» (Marie, 1997: 70). 
du lien religieux doit être nuancée, car ces jeunes s’identifient à leur association ${ }^{36}$. Ainsi, la possibilité d'une "adhésion religieuse [qui] se fait plus fluide, plus nomade», pour se référer à l'ouvrage de René Otayek (2000), ne nous paraît pas si certaine, et nous rejoignons davantage les propos d'Alain Marie: «L'avènement des subjectivités africaines est un processus nécessairement syncrétique, métisse, et donne lieu à des formules de compromis [... entrel'aspiration à l'indépendance et le sentiment profond de recevoir sa pleine identité de ses enracinements communautaires» (1997: 436).

L'enracinement communautaire se construit en lien avec des actions citoyennes qui, comme nous l'avons montré, visent à redéfinir les modalités de participation aux débats de société en cours dans chacun des quatre espaces publics nationaux abordés ici. La «citoyenneté culturelle» revendiquée par ces jeunes, en faisant référence à la umma, renvoie quant à elle à un espace qui déborde le cadre national. Le «bon musulman» est dès lors ancré dans des rapports sociaux concrets et quotidiens tout en participant d'un espace d'identification déterritorialisé construit autour de la communauté universelle des croyants.

Pour autant, ces jeunes parviennent-ils à se construire de solides espaces d'autonomie et de résistance en affirmant plus nettement leur identité islamique, en exprimant leur citoyenneté culturelle? Non, dans la mesure où, quelque soit leur cursus scolaire, leurs espaces d'autonomie demeurent fragiles en raison d'une dégradation des conditions socio-économiques et d'une progression démographique des jeunes. Oui, si l'on tient compte de plusieurs autres faits: ces jeunes ont participé au débat islamique en créant des associations islamiques étudiantes ou de quartier; ils souhaitent garder leur autonomie d'action vis-à-vis d'associations plus anciennes desquelles ils sont toutefois proches idéologiquement ${ }^{37}$; ils exercent aussi leur liberté de parole dans leurs journaux et sur divers postes de radio, et affichent une liberté de ton dans leurs prônes. Le renouveau de l'activisme religieux permet ainsi aux jeunes de s'insérer dans l'espace public, à la fois comme individus mais aussi comme membres de la umma, et de revendiquer leur droit d'expression et d'auto-détermination.

\section{RÉSUMÉ}

La question des jeunes est un élément essentiel pour comprendre les dynamiques de l'espace public ${ }^{38}$ et les pratiques citoyennes qui émergent dans l'Afrique d'aujourd'hui. Leur importance tient non seulement à leur poids démographique imposant, mais aussi à leur transformation, souvent dramatique, en tant qu'acteurs sociaux dans l'espace public africain au cours des années 1990. Cette transformation encourage la création de nouvelles formes de légitimité et de nouveaux espaces d'expression individuelle

36. Par exemple, l'expression « être Ibadou» perdure au Sénégal.

37. L'AEEMB à Ouagadougou se situe dans le sillage du Mouvement Sunnite; l'AEMUD dans celui de la JIR; les associations islamiques de quartier en Côte d'Ivoire sont très près du Conseil national islamique et de la Ligue des prédicateurs de Côte d'Ivoire.

38. Définis comme des espaces d'expression, plus ou moins libres, encourageant l'échange d'idées contradictoires et de discours, mais incluant aussi l'expression non-verbale (voir Diouf, 2003 pour une définition similaire de l'espace public). 
ou collective, et correspond à une mutation radicale de l'idée de citoyenneté, qui fait appel à de nouvelles ressources et qui remodèle les dynamiques nationales d'inclusion et d'exclusion. Ainsi, la constitution de nouveaux espaces d'expression encourage une conception moins restrictive de la participation citoyenne dans la mesure où les jeunes veulent faire entendre leur opinion et participer ouvertement aux divers débats de société. En lien avec les concepts de «citoyenneté culturelle» et de «contre-nation», nous nous proposons dans cet article d'examiner le rôle des jeunes dans le contexte de réaffirmation de l'identité islamique qui a marqué l'Afrique de l'Ouest francophone à partir des années 1980 et plus encore dans les années 1990, particulièrement dans les grands centres urbains du Sénégal, du Mali, du Burkina Faso et de la Côte d'Ivoire. Nous nous penchons plus spécifiquement sur la question du rapport entre jeunes (comme catégorie sociale), religion et espace public.

\section{BIBLIOGRAPHIE}

Abdullah, I. et al. (1997). "Lumpen Youth Culture and Political Violence: The Sierra Leone Civil War", Africa Development, vol. 23, $\mathrm{n}^{\circ}$ 3, p. 171-216.

Alidou, O. (2005). Engaging Modernity: Muslim Women and the Politics of Agency, Madison, University of Wisconsin Press.

Almeida-Topor, H. D' (1992). "“Jeune”, “Jeunes", “Jeunesse": réflexions autour d'une terminologie», in H. D'Almeida-Topor (dir.), Les jeunes en Afrique, t. 1: évolution et rôle (XIX ${ }^{e}-\mathrm{XX} \mathrm{X}^{e}$ siècles), Paris, L'Harmattan, p. 14-16.

Audrain, X. (2004). «Devenir "Baye Fall" pour être soi: le religieux comme vecteur d'émancipation individuelle au Sénégal», Politique Africaine, vol. 94, p. 149-165.

Augis, E. (2005). «Dakar's Sunnite Women: The Politics of Person», in M. Gomez-Perez (dir.), L'islam politique au sud du Sahara: identités, discours et enjeux, Paris, Karthala, p. 309-326.

Augis, E. (2002). Dakar's Sunnite Women: The Politics of Person, Ph.D. Dissertation, University of Chicago.

Brenner, L. (2003). «Youth as Political Actors in Mali», in P. Robinson, C. Newbury et M. Diouf (éd.), Transitions in Africa: Expanding Political Spaces.

Brenner, L. (2001). Controlling Knowledge: Religion, Power and Schooling in a West African Muslim Society, Bloomington, Indiana University Press.

BRENNER, L. (1993a). «La culture arabo-islamique au Mali», in R. OtAYEK (dir.), Le radicalisme islamique au sud du Sahara: Da'wa, arabisation et critique de l'Occident, Paris, Karthala et Talence, M.S.H.A.

Brenner, L. (1993b). «Constructing Muslim Identities in Mali», in L. Brenner (éd.), Muslim Identity and Social Change in Sub-Saharan Africa, London, Hurst, p. 59-78.

Calhoun, C. (2007). Nations Matter: Citizenship, Solidarity and the Cosmopolitan Dream, New York, Routledge.

Cisse, I. (1994). Islam et État au Burkina Faso: de 1960 à 1990, Thèse de doctorat, Université Paris Diderot.

Cisse, I. (1990). "Les médersas au Burkina: l'aide arabe et la croissance d'un système d'enseignement arabo-islamique», Islam et sociétés au sud du Sahara, $\mathrm{n}^{\circ}$ 4, p. 57-72.

Cisse, M. (1985).» Être jeune aujourd'hui», Études Maliennes, vol. 35-36, p. 94-103.

Comaroff, J. et J. Comaroff (2000a). «Réflexions sur la jeunesse: du passé à la postcolonie», Politique Africaine, vol. 8o, décembre, p. 80-109.

Comaroff, J. et J. Comaroff (20oob). «Millennial Capitalism: First Thoughts on a Second Coming», Public Culture, vol. 12, $\mathrm{n}^{\circ}$ 2, p. 291-343.

Comaroff, J. et J. Comaroff (1999). Civil Society and the Political Imagination in Africa: Critical Perspectives, Chicago and London, University of Chicago Press. 
Coulon, C. (1983). Les musulmans et le pouvoir en Afrique noire, Paris, Karthala.

Coulon, C. (1981). Le marabout et le Prince: islam et pouvoir au Sénégal, Paris, Pedone.

Cruise O'Brien, D. (1996). «A Lost Generation? Youth Identity and State Decay in West Africa», in R. Werbner et T. Ranger (éd.), Postcolonial Identities in Africa, London and New Jersey, Zed Books, p. 55-74.

DAO, M. (1991). Le wahhabisme à Ouagadougou de 1964 à 1988, Mémoire de maîtrise, Université de Ouagadougou.

Davis, K. (2002). Preaching to the Converted: Charismatic Leaders, Performances and Electronic Media in Contemporary Islamic Communities, M.Sc. Dissertation, Concordia University.

Diaw, T. (1992). «Les jeunes, la religion, la spiritualité: formes d'encadrement habituelles, nouveaux groupements (sectes), le cas du Sénégal », in H. D’Almeida-Topor (éd.), Les jeunes en Afrique, t. 2 : la politique et la ville, Paris, L'Harmattan, p. 333-346.

Diawara, M. (1996). «Mouvement associatif et transformations du champ politique», in R. Otayek (dir.), Le Burkina entre révolution et démocratie, Paris, Karthala, p. 229-246.

Diop, M.C. et M. Diouf (1990). Le Sénégal sous Abdou Diouf, Paris, Karthala.

Diouf, M. (2003). «Engaging Postcolonial Cultures: African Youth and Public Space», African Studies Review, vol. $46, \mathrm{n}^{\circ}$ 1, p. 1-12.

Diouf, M. et R. Collignon (2001). «Les jeunes du Sud et le temps du monde: identités, conflits et adaptations», Autrepart, vol. 18.

Dolby, N. (2006). «Popular Culture and Public Space in Africa: The Possibilities of Cultural Citizenship », African Studies Review, vol. 49, $\mathrm{n}^{\circ}$ 3, p. 31-47.

El-Kenz, A. (1995). «Les jeunes et la violence», in S. ElLis (dir.), L'Afrique maintenant, Paris, Karthala, p. 87-109.

FALL, M. (1993). «Les arabisants du Sénégal: contre-élites ou courtiers? », in R. OtAYек (dir.), Le radicalisme islamique au sud du Sahara: Da'wa, arabisation et critique de l'Occident, Paris, Karthala, p. 197-212.

FALL, M. (1985). "La question islamique au Sénégal: la religion contre l'État?», Le Mois en Afrique, $\mathrm{n}^{\circ}$ 229-230, p. 37-46.

FALL, M. (1983). "Les dynamiques islamiques au Sénégal», Cultures et développement, vol. XV, ${ }^{\circ} 4$, p. $717-726$.

Gary-Tounkara, D. (2005). «La communauté musulmane et la quête de l'égalité politique dans la Côte d'Ivoire de l'ivoirité (1993-200o)», in M. Gomez-Perez (dir.), L'islam politique au sud du Sahara: identités, discours et enjeux, Paris, Karthala, p. 601-620.

Gomez-Perez, M. (2008). "Autour de mosquées à Ouagadougou et à Dakar: lieux de sociabilité et reconfiguration des communautés musulmanes», in L. Fouchard, O. Goerg et M. Gomez-Perez (dir.), Les lieux de sociabilité en Afrique, Paris, L'Harmattan (sous presse).

Gomez-Perez, M. (2005a). L'islam politique au sud du Sahara: identités, discours et enjeux, Paris, Karthala.

Gomez-Perez, M. (2005b), «Généalogie de l'islam réformiste au Sénégal des années 50 à nos jours: figures, savoirs et réseaux», in A. Mary, L. Fouchard et R. Оtayex (dir.), Entreprises religieuses et réseaux transnationaux en Afrique de l'Ouest, Paris, Karthala, p. 193-222.

Gomez-Perez, M. (1999). «Bilan et perspectives de l'enseignement de l'arabe dans les écoles franco-arabes à Dakar et à Thiès: vers la marginalisation d'une jeunesse?», in M. Gomez-Perez et V. Lacabanne (dir.), La diffusion des savoirs dans les Tiers-Mondes: contraintes et perspectives, Paris, Publications Universitaires Denis Diderot, p. 41-69.

Gomez-Perez, M. (1997). Une histoire des associations islamiques sénégalaises (Saint-Louis, Dakar, Thiès): itinéraires, stratégies et prises de parole (1930-1993), Thèse de doctorat, Université Paris Diderot, 2 tomes.

Gomez-Perez, M. (1994). «L'islamisme à Dakar: d'un contrôle social total à une culture du pouvoir?», Afrika Spectrum, $\mathrm{n}^{\circ}$ 29, p. 79-98.

Gomez-Perez, M. (1990). «L'affaire des “versets sataniques” au Sénégal. Dossier de presse», Islam et sociétés au sud du Sahara, $\mathrm{n}^{\circ}$ 4, p. 173-177. 
Gomez-Perez, M., M.-N. LeBlanc et S. Savadogo (2007). «Youth and Islam in the 1990s: Re-Thinking an Inter-Generational Perspective», communication, AEGIS, Leiden, Juillet.

HAGBERG, S. (2002). "Enough is Enough": An Ethnography of the Struggle Against Impunity in Burkina Faso ", Journal of Modern African Studies, vol. 40, n 2, p. 217-246.

Hamel, J. (1999). «La jeunesse n'est pas un mot... Petit essai d'épistémologie pratique», in M. Gauthier, J.-F. Guillaume (dir.), Définir la jeunesse? D'un bout à l'autre de monde, Paris/Montréal, L'Harmattan/ IQRC, p. 22-44.

Hervieu-Léger, D. (1990). De l'émotion en religion: renouveaux et traditions, Paris, Centurion.

Hilgers, M. et J. Mazzocchetti (2006). «L'après-Zongo: entre ouverture politique et fermeture des possibles», Politique Africaine, vol. 101, mars-avril, p. 5-18.

IhLe, A.H. (2003). "It's All about Morals." Islam and Social Mobility among Young and Committed Muslims in Tamale, Northern Ghana, Ph.D. dissertation, Copenhagen University.

Janson, M. (2007). «Appropriating Islam: The Tensions between "Traditionalists" and "Modernists" in The Gambia ", Islam et sociétés au sud du Sahara, vol. 1, p. 61-79.

JuA, N. (2003). «Differential Responses to Disappearing Transitional Pathways: Redefining Possibility among Cameroonian Youths", African Studies Review, vol. 46, n ${ }^{\circ}$, p. 13-36.

KABA, L. (1974). The Wahhabiyya, Evanston, Northwestern University Press.

Kane, O. (2003). Muslim Modernity in Postcolonial Nigeria, Leiden, Brill.

Khilnani, S. (2001). «The Development of Civil Society», in S. Kaviraj et S. Khilnani (dir.), Civil Society: History and Possibilities, Cambridge, Cambridge University Press, p. 11-32.

KiefFer, J. (2006). «Les jeunes des "grins" de thé et la campagne électorale à Ouagadougou», Politique Africaine, vol. 101, mars-avril, p. 63-82.

Konate, Y. (2003). «Les enfants de la balle: de la FESCI aux mouvements des patriotes», Politique Africaine, vol. 89, p. 49-70.

Kone-DAO, M. (2005). «Implantation et influence du wahhabisme au Burkina Faso de 1963 à 2002», in M. Gomez-Perez (dir.), L'islam politique au sud du Sahara: identités, discours et enjeux, Paris, Karthala, p. 449-459.

KouAndA, A. (1996). «La lutte pour l'occupation et le contrôle des espaces réservés aux cultes à Ouagadougou », in R. Отауек et al. (dir.), Le Burkina entre révolution et démocratie (1983-1993), Paris, Karthala, p. 91-99.

KouAndA, A. (1989). «Les conflits au sein de la communauté musulmane du Burkina: 1962-1986», Islam et sociétés au sud du Sahara, vol. 3, mai, p. 7-26.

Last, M. (1992). «The Power of Youth, Youth of Power», in H. D’Almeida-Topor (dir.), Les jeunes en Afrique, t. 2 : la politique et la ville, Paris, L'Harmattan, p. 375-399.

LAurent, P.-J. (2003). Les pentecôtistes du Burkina Faso: mariage, pouvoir et guérison, Paris, Karthala.

LeBLAnC, M.-N. (2008). «Nouveaux regards sur la vie des jeunes musulmanes en Côte d'Ivoire: dynamiques de sociabilité chez les jeunes arabisantes au tournant du XxI ${ }^{e}$ siècle», in L. Fouchard, O. Goerg et M. Gomez-Perez, Les lieux de sociabilité en Afrique, Paris, L'Harmattan (sous presse).

LeBlanc, M.-N. (2007). «Imaniya and Young Muslim Women in Côte d'Ivoire», Anthropologica, vol. 49, $\mathrm{n}^{\circ}$ 1, p. 35-50.

LeBlanc, M.-N. (2006). «De la tradition à l'islam: l'orthodoxie à l'encontre des rites culturels», Cahiers des Études Africaines, vol. 182, nº 2, p. 417-436.

LeBlanc, M.-N. (2005). «Hadj et changements identitaires: les jeunes musulmans d'Abidjan et de Bouaké, en Côte d'Ivoire, dans les années 1990 », in M. Gomez-Perez (dir.), L'islam politique au sud du Sahara: identités, discours, enjeux, Paris, Karthala, p. 131-157.

LeBlanc, M.-N. (2003). «Les trajectoires de conversion et l'identité sociale chez les jeunes dans le contexte postcolonial ouest-africain: les jeunes musulmans et les jeunes chrétiens en Côte d'Ivoire», Anthropologie et Sociétés, vol. 27, $\mathrm{n}^{\circ}$ 1, p. 85-110.

LeBlanc, M.-N. (2000). «Fashion and the Politics of Identity: Versioning Womanhood and Muslimhood in the Face of Tradition and Modernity», Africa, vol. 70, $\mathrm{n}^{\circ}$ 3, p. 443-481. 
LeBlanc, M.-N. (1999). «The Production of Islamic Identities through Knowledge Claims in Bouaké, Côte d'Ivoire», African Affairs, vol. 98, n 393, p. 485-508.

LeBlanc, M.-N. (1998). Youth, Islam and Changing Identities in Bouaké, Côte d'Ivoire, Ph.D. dissertation, University College.

LoadA, A. (2006). «L'élection présidentielle du 13 novembre 2005: un plébiscite par défaut», Politique Africaine, vol. 101, mars-avril, p. 19-41.

Loada, A. (1999). «Réflexions sur la société civile en Afrique: le Burkina de l'après-Zongo ", Politique Africaine, vol. 76, décembre, p. 136-150.

LOADA, A. (1996). «Blaise Compaoré ou l'architecte d'un nouvel ordre politique», in R. ОтAYeK et al. (dir.), Le Burkina entre révolution et démocratie, Paris, Karthala, p. 277-297.

Loimeier, R. (1997). Islamic Reform and Political Change in Northern Nigeria, Chicago, Northwestern University Press.

Magassouba, M. (1985). L'islam au Sénégal, demain les mollahs?, Paris, Karthala.

Marie, A. (1997). L'Afrique des individus, Paris, Karthala.

Marshall-Fratani, R. (2006). «The War of "Who Is Who": Autochtony, Nationalism, and Citizenship in the Ivorian Crisis", African Studies Review, vol. 49, n 2, p. 9-43.

Marshall-Fratani, R et D. Péclard (2002). «La religion du sujet en Afrique», Politique Africaine, «Les sujets de Dieu", vol. 87, p. 5-19.

Masquelier, A. (2001). Prayer Has Spoiled Everything: Possession, Power, and Identity in an Islamic Town in Niger, Durham, Duke University Press.

Maxwell, D. (2001). «"Sacred History, Social History”: Traditions and Texts in the Making of a Southern African Transnational Religious Movement ", Comparative Studies in History and Society, vol. 43, $\mathrm{n}^{\circ} \mathrm{3}$, p. 502-524.

Meyer, B. (1998). «Make a Break with the Past: Memory and Postcolonial Modernity in Ghanaian Pentecostal Discourse», in R. Werbner (dir.), Memory and the Post-Colony: African Anthropology and the Critique of Power, London and New York, Routledge.

Miller, T. (1998). Technologies of Truth: Cultural Citizenship and the Popular Media, Minneapolis, University of Minnesota Press.

Miran, M. (2006). Islam, histoire et modernité en Côte d'Ivoire, Paris, Karthala.

Miran, M. (2000). «Vers un nouveau prosélytisme islamique en Côte d'Ivoire: une révolution discrète», Autrepart, vol. 16, p. 139-160.

Miran, M. (1998). «Le wahhabisme à Abidjan: dynamisme urbain d'un islam réformiste en Côte d'Ivoire contemporaine (1960-1996)", Islam et sociétés au sud du Sahara, vol. 12, p. 5-74.

Otayeк, R. (2000). Identité et démocratie dans un monde global, Paris, Presses de Science Po.

Отауек, R. (1996a). «L'islam et la révolution au Burkina Faso: mobilisation politique et reconstruction identitaire», Social Compass, vol. 43, n² 2, p. 233-247.

OtAYeк, R. (1996b). «Introduction: du Burkina du changement social et de la démocratie», in R. ОтАуек et al. (dir.), Le Burkina entre révolution et démocratie, Paris, Karthala, p. 7-19.

Отауек, R. (1993a). «Introduction: des nouveaux intellectuels musulmans d'Afrique noire», in R. Отауек (dir.), Le radicalisme islamique au sud du Sahara: Da'wa, arabisation et critique de l'Occident, Paris, Karthala.

OtaYeк, R. (1993b). «L'affirmation élitaire des arabisants au Burkina Faso : enjeux et contradictions», in R. Отауек (dir.), Le radicalisme islamique au sud du Sahara: Da'wa, arabisation et critique de l'Occident, Paris, Karthala, p. 229-252.

Oтауек, R. (1984). «La crise de la communauté musulmane de Haute-Volta: l'islam voltaïque entre réformisme et tradition, autonomie et subordination», Cahiers d'études africaines, vol. $24, \mathrm{n}^{\circ} 3$, p. 299-320.

Otayek, R. et D. Diallo (1998). «Dynamisme protestant, développement participatif et démocratie locale: le cas de l'Office de développement des Églises évangéliques (Burkina Faso) ", Afrique contemporaine, $1^{\text {er }}$ trimestre. 
Richards, P. (1996). Fighting for the Rain Forest: War, Youth and resources in Sierra Leone, Oxford, James Currey.

Roy, O. (1999). «Le post-islamisme», Revue des mondes musulmans et de la Méditerranée, n 85-86, p. 11-30.

SAmson, F. (2005). Les marabouts de l'islam politique: le Dahiratoul Moustarchidina Wal Moustarchitady, un mouvement néo-confrérique sénégalais, Paris, Karthala.

Savadogo, B.M. et A. Sylla (2006). "Les mosquées comme espaces de sociabilité des communautés musulmanes des lieux de détresse d'Abidjan ", communication, Conférence internationale organisée par le SEDET et le CEAN, Les lieux de sociabilité urbaine dans la longue durée en Afrique, Paris, 22 24 juin.

Savadogo, B.M. (2005). "L'intervention des associations musulmanes dans le champ politique en Côte d'Ivoire depuis 1990", in M. Gomez-Perez (dir.), L'islam politique au sud du Sahara: identités, discours et enjeux, Paris, Karthala, p. 583-600.

Schulz, D. (2006). «Promises of (Im)mediate Salvation: Islam, Broadcast Media, and the Remaking of Religious Experience in Mali», American Ethnologist, vol. 33, n 2, p. $210-229$.

Schulz, D. (2003). «Charisma and Brotherhood Revisited: Mass-Mediated Forms of Spirituality in Urban Mali», Journal of Religion in Africa, vol. 33, $\mathrm{n}^{\circ}$ 2, p. 146-171.

SoAres, B.F. (2005). Islam and the Prayer Economy: History and Authority in a Malian Town, Ann Arbor, University of Michigan Press.

Soares, B.F. (2004). «Islam and Public Piety in Mali», in A. Salvatore et D.F. Eickelman (dir.), Public Islam and the Common Good, Leiden, Brill.

Souley, H. (2002). La presse africaine et les événements du 11 septembre, Aix-en-Provence, Institut d'études africaines.

Tozy, M. (1995). «Les mouvements de renouveau religieux», in S. Ellis (dir.), L'Afrique maintenant, Paris, Karthala.

Traoré, B. (2005). «Islam et politique à Bobo-Dioulasso de 1940 à 2002", in M. Gomez-Perez (dir.), L'islam politique au sud du Sahara: identités, discours et enjeux, Paris, Karthala, p. 417-447.

Umar, M.S. (1988). Sufism and Anti-Sufism in Nigeria, M.A. Dissertation, University of Kano.

Villalon, L.A. (1999). «Generational Changes, Political Stagnation, and the Evolving Dynamics of Religion and Politics in Senegal», Africa Today, vol. 46, $\mathrm{n}^{\circ} 3-4$, p. 130-147.

WIse, C. (1998). «Chronicle of a Student Strike in Africa: The Case of Burkina Faso, 1996-1997», African Studies Review, vol. 41, n 2, p. 19-36. 